\title{
Period-halving Bifurcation of a Neuronal Recurrence Equation
}

\author{
René Ndoundam ${ }^{a, b}$ \\ ${ }^{a}$ University of Yaounde I, UMI 209, UMMISCO, P.o.Box 337 Yaounde, \\ Cameroon \\ ${ }^{b}$ University of Yaounde I, LIRIMA, Team GRIMCAPE, Faculty of Science, \\ Department of Computer Science, P.o. Box 812 Yaounde, Cameroon \\ E.mail : ndoundam@gmail.com ,ndoundam@yahoo.com
}

\begin{abstract}
We study the sequences generated by neuronal recurrence equations of the form $x(n)=\mathbf{1}\left[\sum_{j=1}^{h} a_{j} x(n-j)-\theta\right]$. From a neuronal recurrence equation of memory size $h$ which describes a cycle of length $\rho(m) \times$ $\operatorname{lcm}\left(p_{0}, p_{1}, \ldots, p_{-1+\rho(m)}\right)$, we construct a set of $\rho(m)$ neuronal recurrence equations whose dynamics describe respectively the transient of length $O\left(\rho(m) \times l c m\left(p_{0}, \ldots, p_{d}\right)\right)$ and the cycle of length $O\left(\rho(m) \times l c m\left(p_{d+1}, \ldots, p_{-1+\rho(m)}\right)\right)$ if $0 \leq d \leq-2+\rho(m)$ and 1 if $d=\rho(m)-1$. This result shows the exponential time of the convergence of neuronal recurrence equation to fixed points and the existence of the period-halving bifurcation.
\end{abstract}

Keywords. Neuronal recurrence equation, cycle length, period-halving bifurcation. 


\section{Introduction}

Caianiello and De Luca [3] have suggested that the dynamic behavior of a single neuron with a memory, which does not interact with other neurons can be modeled by the following recurrence equation :

$$
x(n)=\mathbf{1}\left[\sum_{j=1}^{k} a_{j} x(n-j)-\theta\right]
$$

where :

- $x(n)$ is a variable representing the state of the neuron at $t=n$.

- $x(0), x(1), \cdots, x(k-2), x(k-1)$ are the initial states.

- $k$ is the memory length, i.e., the state of the neuron at time $t=n$ depends on the states $x(n-1), \ldots, x(n-k)$ assumed by the neuron at the $k$ previous steps $t=n-1, \ldots, n-k$.

- $a_{j}(j=1, \ldots, k)$ are real numbers called the weighting coefficients. More precisely, $a_{j}$ represents the influence of the state of the neuron at time $n-j$ on the state assumed by the neuron at time $n$.

- $\theta$ is a real number called the threshold.

- $\mathbf{1}[u]=0$ if $u<0$, and $\mathbf{1}[u]=1$ if $u \geq 0$.

The system obtained by interconnecting several neurons is called a neural network. These networks were introduced by McCulloch and Pitts 7, and are quite powerful. Neural networks are able to simulate any sequential machine or Turing machine if an infinite number of cells is provided. Neural networks have been studied extensively as tools for solving various problems such as classification, speech recognition, and image processing [19]. The field of application of threshold functions is large [1, 9, 10, 19]. The spin moment of the spin glass system is one of the most cited example in solid state physics that has been 
simulated by neural networks.

Neural networks are usually implemented by using electronic components or is simulated in software on a digital computer. One way in which the collective properties of a neural network may be used to implement a computational task is by way of the concept of energy minimization. The Hopfield network is a well-known example of such an approach. It has attracted great attention in literature as a content-addressable memory [2].

Given a finite neural network, the configuration assumed by the system at time $\mathrm{t}$ is ultimately periodic. As a consequence, there is an integer $p>0$ called the period (or a length of a cycle) and another integer $T \geq 0$ called the transient length such that:

- $Y(p+T)=Y(T)$

- $\exists T^{\prime}$ and $p^{\prime}\left(T^{\prime}, p^{\prime}\right) \neq(T, p) \quad T \geq T^{\prime}$ and $p \geq p^{\prime} \quad$ such that $Y\left(p^{\prime}+T^{\prime}\right)=$ $Y\left(T^{\prime}\right)$

where $Y(t)=(x(t), x(t-1), \ldots, x(t-k+2), x(t-k+1))$. The period and the transient length of the sequences generated are good measures of the complexity of the neuron. A bifurcation occurs when a small smooth change made to the parameter values (the bifurcation parameters) of a system causes a sudden 'qualitative' or topological change in its behaviour. A period halving bifurcation in a dynamical system is a bifurcation in which the system switches to a new behaviour with half the period of the original system. A great variety of results have been established on recurrence equations modeling neurons with memory 1, 4, 15, 6, 11, 12, 14, 15, 20. However some mathematical properties are still very intriguing and many problems are being posed. For example, the question remains as to whether there exists one neuronal recurrence equation with transients of exponential lengths [18]. In [13], we give a positive answer to this question by exhibiting a neuronal recurrence equation with memory which generates a sequence of exponential transient length and exponential period length 
with respect to the memory length. Despite this positive answer, one question remains: does there exist one neuronal recurrence equation with exponential transient length and fixed point?

In this work, from a neuronal recurrence equation of memory size $(6 m-1) \times$ $(\rho(m))^{2}$, whose dynamics contains a cycle of length $\rho(m) \times l c m\left(p_{0}, p_{1}, \ldots, p_{-1+\rho(m)}\right)$, we build a set of $\rho(m)$ neuronal recurrence equations whose dynamics describe respectively:

- the transient of length $\rho(m) \times l c m\left(p_{0}, p_{1}, \ldots, p_{d}\right)+h+d+1-(\rho(m) \times$ $\left.\left(1+p_{d}\right)\right)$, if $0 \leq d \leq-1+\rho(m)$

- the cycle of length $\rho(m) \times l c m\left(p_{d+1}, p_{d+2}, \ldots, p_{-1+\rho(m)}\right)$ if $0 \leq d \leq-2+$ $\rho(m)$ and 1 if $d=\rho(m)-1$

Thus, we give a positive answer to the precedent question.

The technique used in this paper to get the period-halving bifurcation is to modify some parameters (weighting coefficients and threshold) of the neuronal recurrence equation. This technique relies on control theory. Controllability is related to the possibility of forcing the system into a particular state by using an appropriate control signal.

The paper is organized as follows: in Section 2, some previous results are presented. Section 3 presents some preliminaries. Section 4 is devoted to the construction of neuronal recurrence equation $z(n, d)$. Section 5 deals with the behavior of neuronal recurrence equation $z(n, d)$. Concluding remarks are stated in Section 6.

\section{Previous Results}

The only study of bifurcation was done by Cosnard and Goles in [6]. Cosnard and Goles [6] studied the bifurcation in two particular cases of neuronal recur- 
rence equation:

Case 1: geometric coefficients and bounded memory

Cosnard and Goles described completely the structure of the bifurcation of the following equation:

$$
x_{n+1}=\mathbf{1}\left[\theta-\sum_{i=0}^{k-1} b^{i} x_{n-i}\right]
$$

when $\theta$ varies. They showed that the associated rotation number is an increasing number of the parameter $\theta$.

Case 2: geometric coefficients and unbounded memory

Cosnard and Goles described completely the structure of the bifurcation of the following equation:

$$
x_{n+1}=\mathbf{1}\left[\theta-\sum_{i=0}^{n} b^{i} x_{n-i}\right]
$$

when $\theta$ varies. They showed that the associated rotation number is a devil's staircase.

From line 11 to line 15 of page 15 in [5], it is written: "This shows that, if there is a neuronal recurrence equation with memory length $k$ that generates sequences of periods $p_{1}, \ldots, p_{r}$, then there is a neuronal recurrence equation with memory length $k r$ that generates a sequence of period $l c m\left(p_{1}, \ldots, p_{r}\right) r$, where $l \mathrm{~cm}$ denotes the least common multiple." This allows us to write the following fundamental lemma of composition of a neuronal recurrence equation:

\section{Lemma 1 [5]}

If there is a neuronal recurrence equation with memory length $k$ that generates sequences of periods $p_{1}, p_{2}, \ldots, p_{r}$, then there is a neuronal recurrence equation with memory length $k r$ that generates a sequence of period $r \cdot l c m\left(p_{1}, \cdots, p_{r}\right)$.

Lemma 1 does not take into account the study of the transient length. One can amend Lemma 1 to obtain the following lemma: 
Lemma 2 [13, 14] If there is a neuronal recurrence equation with memory length $k$ that generates a sequence $\left\{x^{\jmath}(n): n \geq 0\right\}, 1 \leq \jmath \leq g$ of transient length $T_{\jmath}$ and of period $p_{\jmath}$ then there is a neuronal recurrence equation with memory length $\mathrm{kg}$ that generates a sequence of transient length $g \cdot \max \left(T_{1}, T_{2}, \ldots, T_{g}\right)$ and of period $g \cdot \operatorname{lcm}\left(p_{1}, p_{2}, \ldots, p_{g}\right)$.

In the following example, we will show that Lemma 1 and Lemma 2 are incomplete.

\section{Example 1:}

Let us suppose that the neuronal recurrence equation defined by Equation (10) generated six sequences

$$
\left\{x^{i}(n): n \geq 0\right\}, 0 \leq i \leq 5
$$

of periods

$$
p_{i}=1,0 \leq i \leq 5
$$

It is clear that each sequence defined by Equation (2) is a fixed point. We present two different cases of evolution.

\section{First case:}

We suppose that

$$
\begin{array}{r}
x^{2 i}(n)=0 ; \forall n, i \text { such that } n \geq 0 \text { and } 0 \leq i \leq 2 \\
x^{2 i+1}(n)=1 ; \forall n, i \text { such that } n \geq 0 \text { and } 0 \leq i \leq 2
\end{array}
$$

It is easy to verify that the shuffle of the neuronal recurrence equation defined by Equations (4) and (5) is

$$
\begin{aligned}
& x^{0}(0) x^{1}(0) \ldots x^{5}(0) x^{0}(1) x^{1}(1) \ldots x^{5}(1) \cdots x^{0}(i) x^{1}(i) \ldots x^{5}(i) \cdots= \\
& 010101010101010101010101 \cdots 010101010101 \cdots
\end{aligned}
$$

The sequence defined by Equation (6) describes a period of length 2. By application of the Lemma 10 the period of the sequence defined by Equation (6) 
should be 6 ( more precisely $6 \times \operatorname{lcm}(1,1,1,1,1,1))$.

\section{Second case:}

We suppose that

$$
\begin{aligned}
& x^{i}(n)=0, \forall n, i \text { such that } n \geq 0 \text { and } i \in\{0,1,3,4\} \\
& x^{i}(n)=1, \forall n, i \text { such that } n \geq 0 \text { and } i \in\{2,5\}
\end{aligned}
$$

It is easy to verify that the shuffle of the neuronal recurrence equation defined by Equations (7) and (8) is

$$
\begin{aligned}
& x^{0}(0) x^{1}(0) \ldots x^{5}(0) x^{0}(1) x^{1}(1) \ldots x^{5}(1) \cdots x^{0}(i) x^{1}(i) \ldots x^{5}(i) \cdots= \\
& 001001001001001001001001001001001001 \cdots 001001 \cdots
\end{aligned}
$$

The sequence defined by Equation (9) describes a period of length 3. By application of the Lemma 1 the period of the sequence defined by Equation (9) should be 6 ( more precisely $6 \times \operatorname{lcm}(1,1,1,1,1,1)$ ).

The first case and the second case of example 1 show that Lemma 1 and Lemma 2 don't take into account all the cases.

One can amend the Lemma 1 as follows:

Lemma 3 If there is a neuronal recurrence equation with memory length $k$ that generates $r$ sequences of periods $p_{1}, p_{2}, \ldots, p_{r}$, then there is a neuronal recurrence equation with memory length $k r$ that generates a sequence of period Per. Per is defined as follows:

First case: $\exists j, 1 \leq j \leq r$ such that $p_{j} \geq 2$

$$
\text { Per }=r \times l c m\left(p_{1}, \cdots, p_{r}\right) \text {. }
$$

Second case: $p_{j}=1 ; \forall j, 1 \leq j \leq r$.

Per is a divisor of $r$. 
The improvement of Lemma 1 doesn't modify all the main results about periods obtained in the papers [5, 11, 12, 14, 15, because all these main results consider only the case where the periods $p_{1}, p_{2}, \ldots, p_{r}$ of the $r$ sequences are greater or equal to 2 .

We can also amend the Lemma 2 as follows:

Lemma 4 If there is a neuronal recurrence equation with memory length $k$ that generates a sequence $\left\{x^{\jmath}(n): n \geq 0\right\}, 1 \leq \jmath \leq g$ of transient length $T_{\jmath}$ and of period $p_{J}$ then there is a neuronal recurrence equation with memory length $\mathrm{kg}$ that generates a sequence of transient length $g \cdot \max \left(T_{1}, T_{2}, \ldots, T_{g}\right)$ and of period Per. Per is defined as follows:

First case: $\exists j, 1 \leq j \leq r$ such that $p_{j} \geq 2$

$$
\text { Per }=r \times \operatorname{lcm}\left(p_{1}, \cdots, p_{g}\right) \text {. }
$$

Second case: $p_{j}=1 ; \forall j, 1 \leq j \leq r$.

$$
\text { Per is a divisor of } g \text {. }
$$

\section{Preliminaries}

Let $k$ be a positive integer. For a vector $a \in \mathbb{R}^{k}$, a real number $\theta \in \mathbb{R}$ and a vector $\phi \in\{0,1\}^{k}$. We define the sequence $\{x(n): n \in \mathbb{N}\}$ by the following recurrence:

$$
x(t)= \begin{cases}\phi(t) ; & t \in\{0, \ldots, k-1\} \\ \mathbf{1}\left(\sum_{i=1}^{k} a_{i} x(t-i)-\theta\right) ; & t \geq k\end{cases}
$$

We denote by $S(a, \theta, \phi)$ the sequence generated by equation (10), $\operatorname{Per}(a, \theta, \phi)$ its period and $\operatorname{Tr} a(a, \theta, \phi)$ its transient length. 
Let $m$ be a positive integer, we denote the cardinality of the set $\mathcal{P}=\{p$ : $p$ prime and $2 m<p<3 m\}$ by $\rho(m)$. Let us denote by $p_{0}, p_{1}, \ldots, p_{-1+\rho(m)}$ the prime numbers belonging to the set $\{2 m+1,2 m+2, \ldots, 3 m-2,3 m-1\}$, the sequence $\left\{\alpha_{i}: 0 \leq i \leq-1+\rho(m)\right\}$ is defined as $\alpha_{i}=3 m-p_{i}, 0 \leq i \leq$ $-1+\rho(m)$.

We also suppose that:

$$
p_{-1+\rho(m)}<p_{-2+\rho(m)}<\cdots<p_{i+1}<p_{i}<\cdots<p_{1}<p_{0}
$$

Subsequently, we consider only the integers $m$ such that $\rho(m) \geq 2$.

It is easy to check that $\{2 m+1,2 m+2, \ldots, 3 m-2,3 m-1\}$ contains at most $\left\lceil\frac{m-1}{2}\right\rceil$ odd integers. It follows that

$$
\rho(m) \leq\left\lceil\frac{m-1}{2}\right\rceil
$$

We set $k=(6 m-1) \rho(m)$ and $\forall i \in \mathbb{N}, 0 \leq i \leq-1+\rho(m)$, we define :

$$
\begin{aligned}
& \mu\left(m, \alpha_{i}\right)=\left\lfloor\frac{k}{3 m-\alpha_{i}}\right\rfloor \\
& \beta\left(m, \alpha_{i}\right)=k-\left(\left(3 m-\alpha_{i}\right) \mu\left(m, \alpha_{i}\right)\right)
\end{aligned}
$$

From the previous definitions, we have $k=\left(\left(3 m-\alpha_{i}\right) \mu\left(m, \alpha_{i}\right)\right)+\beta\left(m, \alpha_{i}\right)$.

It is clear that $\forall i \in \mathbb{N}, 0 \leq i \leq-1+\rho(m)$

$$
2 m+1 \leq 3 m-\alpha_{i} \leq 3 m-1
$$

This implies that

$$
\frac{(6 m-1) \rho(m)}{3 m-1} \leq \frac{k}{3 m-\alpha_{i}} \leq \frac{(6 m-1) \rho(m)}{2 m+1}
$$

Therefore

$$
2 \rho(m) \leq \mu\left(m, \alpha_{i}\right) \leq 3 \rho(m)
$$

$\forall i \in \mathbb{N}, 0 \leq i \leq-1+\rho(m)$, we want to construct a neuronal recurrence equation $\left\{x^{\alpha_{i}}(n): n \geq 0\right\}$ with memory of length $k$ which evolves as follows :

$$
\underbrace{00 \ldots 0}_{\beta\left(m, \alpha_{i}\right)} \underbrace{100 \ldots 0}_{3 m-\alpha_{i}} \underbrace{100 \ldots 0}_{3 m-\alpha_{i}} \cdots \underbrace{100 \ldots 0}_{3 m-\alpha_{i}} \cdots \underbrace{100 \ldots 0}_{3 m-\alpha_{i}} \cdots
$$


and which describes a cycle of length $3 m-\alpha_{i}=p_{i}$.

$\forall i \in \mathbb{N}, 0 \leq i \leq-1+\rho(m)$, let $\phi^{\alpha_{i}} \in\{0,1\}^{k}$ be the vector defined by

$$
\phi^{\alpha_{i}}(0) \ldots \phi^{\alpha_{i}}(k-1)=\underbrace{0 \ldots 0}_{\beta\left(m, \alpha_{i}\right)} \underbrace{10 \ldots 0}_{\mu\left(m, \alpha_{i}\right) p_{i}} \cdots \underbrace{10 \ldots 0}_{p_{i}}
$$

In other words, $\phi^{\alpha_{i}}$ is defined by:

$$
\phi^{\alpha_{i}}(j)= \begin{cases}1 & \text { if } \exists \ell, 0 \leq \ell \leq \mu\left(m, \alpha_{i}\right)-1 \text { such that } j=\beta\left(m, \alpha_{i}\right)+\ell p_{i} \\ 0 & \text { otherwise }\end{cases}
$$

We define the neuronal recurrence equation $\left\{x^{\alpha_{i}}(n): n \geq 0\right\}$ by the following recurrence:

$$
x^{\alpha_{i}}(t)= \begin{cases}\phi^{\alpha_{i}}(t) & ; t \in\{0, \ldots, k-1\} \\ \mathbf{1}\left(\sum_{j=1}^{k} \bar{a}_{j} x^{\alpha_{i}}(t-j)-\bar{\theta}\right) & ; t \geq k\end{cases}
$$

where $\bar{a}_{j}$ is defined as follows:

First case: $\rho(m)$ is even and $\forall i_{2} \in \mathbb{N}, 0 \leq i_{2} \leq-1+\rho(m)$

$$
\bar{a}_{j}= \begin{cases}2 & \text { if } j \in \operatorname{Pos}\left(\alpha_{i_{2}}\right) \text { and } j \leq \frac{3 \times \rho(m) \times p_{i_{2}}}{2} \\ -2 & \text { if } j \in \operatorname{Pos}\left(\alpha_{i_{2}}\right) \text { and } j>\frac{3 \times \rho(m) \times p_{i_{2}}}{2} \\ 0 & \text { otherwise. }\end{cases}
$$

Second case: $\rho(m)$ is odd, $\rho(m) \geq 3$ and $\forall i_{2} \in \mathbb{N}, 0 \leq i_{2} \leq-1+\rho(m)$

$$
\bar{a}_{j}= \begin{cases}2 & \text { if } j \in \operatorname{Pos}\left(\alpha_{i_{2}}\right) \text { and } j \leq \frac{(3 \rho(m)-1)}{2} \times p_{i_{2}}, \\ -2 & \text { if } j \in \operatorname{Pos}\left(\alpha_{i_{2}}\right) \text { and } \frac{(3 \rho(m)+1)}{2} \times p_{i_{2}} \leq j \leq(2 \rho(m)-2) \times p_{i_{2}}, \\ -1 & \text { if } j \in\left\{(2 \rho(m)-1) \times p_{i_{2}}, 2 \rho(m) \times p_{i_{2}}\right\} \\ 0 & \text { otherwise. }\end{cases}
$$


We also define:

$$
\begin{aligned}
\operatorname{Pos}\left(\alpha_{i}\right) & =\left\{j p_{i}: j=1, \ldots, 2 \rho(m)\right\} \\
& =\left\{p_{i}, 2 p_{i}, \ldots,(-1+2 \rho(m)) p_{i}, 2 \rho(m) p_{i}\right\}, \quad 0 \leq i \leq-1+\rho(m) \\
D & =\{i: i=1, \ldots, k\}=\{1,2, \ldots, k-1, k\} \\
F & =\bigcup_{i=0}^{-1+\rho(m)} \operatorname{Pos}\left(\alpha_{i}\right) \\
G & =D \backslash F \\
\bar{\theta} & =2 \times \rho(m) \\
k & =(6 m-1) \times \rho(m)
\end{aligned}
$$

By definition $\operatorname{Pos}\left(\alpha_{i}\right)$ represents the set of indices $j, 1 \leq j \leq k$ such that $x^{\alpha_{i}}(k-j)=1$.

From the definition of $\operatorname{Pos}\left(\alpha_{i}\right)$ and from Equation (15), one can easily verify that

$$
\begin{aligned}
j \in \operatorname{Pos}\left(\alpha_{i}\right) & \Longrightarrow x^{\alpha_{i}}(k-j)=1 \\
j \in D \backslash \operatorname{Pos}\left(\alpha_{i}\right) & \Longrightarrow x^{\alpha_{i}}(k-j)=0
\end{aligned}
$$

$\forall d \in \mathbb{N}, 0<d<p_{i}$, we also denote $\operatorname{PPos}\left(\alpha_{i}, d\right)$ the set of indices $j$ such that $x^{\alpha_{i}}(k+d-j)=1$, in other words:

$$
\operatorname{PPos}\left(\alpha_{i}, d\right)=\left\{j: x^{\alpha_{i}}(k+d-j)=1 \text { and } 1 \leq j \leq k\right\}
$$

$\forall i, d \in \mathbb{N}, 0 \leq i \leq-1+\rho(m)$ and $0<d<p_{i}$, we denote:

$$
\begin{aligned}
& Q\left(\alpha_{i}, d\right)=\left\{d+j p_{i}: j=0,1, \ldots, \mu\left(m, \alpha_{i}\right)\right\}, \quad 0<d \leq \beta\left(m, \alpha_{i}\right) \\
& Q\left(\alpha_{i}, d\right)=\left\{d+j p_{i}: j=0,1, \ldots,-1+\mu\left(m, \alpha_{i}\right)\right\}, \quad \beta\left(m, \alpha_{i}\right)<d<p_{i} \\
& E\left(\alpha_{i}, d\right)=Q\left(\alpha_{i}, d\right) \cap F
\end{aligned}
$$

The neuronal recurrence equation $\left\{x^{\alpha_{i}}(n): n \geq 0\right\}$ with memory of length $k$ is defined by Equations (15) and (16). 
We will show that the neuronal recurrence equation $\left\{x^{\alpha_{i}}(n): n \geq 0\right\}$ evolves as specified in equation (14).

In the following proposition, we present an important property.

Proposition 1 [14] $\forall i \in \mathbb{N}, 0 \leq i \leq-1+\rho(m)$ and $\forall d \in \mathbb{N}, 1 \leq d<p_{i}$

$$
\operatorname{card} E\left(\alpha_{i}, d\right) \leq \rho(m)-1
$$

The following proposition characterizes the sum of the interaction coefficients $\bar{a}_{j}$ when $j \in \operatorname{Pos}\left(\alpha_{i}\right)$

Proposition $2 \forall i \in \mathbb{N}, 0 \leq i \leq-1+\rho(m)$, we have

$$
\sum_{j \in \operatorname{Pos}\left(\alpha_{i}\right)} \bar{a}_{j}=2 \times \rho(m) .
$$

The following lemma characterizes the evolution of the sequence $\left\{x^{\alpha_{i}}(n)\right.$ : $n \geq 0\}$ at time $t=k$.

\section{Lemma 5}

$$
x^{\alpha_{i}}(k)=1 .
$$

From Lemma 5 and Equation (15), it is easy to verify that

$$
\operatorname{PPos}\left(\alpha_{i}, 1\right)=Q\left(\alpha_{i}, 1\right)
$$

From the definition of $E\left(\alpha_{i}, 1\right)$, from Equation (15), from Equation (28) and from the Lemma 5 , we check easily that:

$$
\ell \in D \backslash E\left(\alpha_{i}, 1\right) \Longrightarrow x^{\alpha_{i}}(k+1-\ell)=0 \text { or } \bar{a}_{\ell}=0 .
$$

The values of the sequence $\left\{x^{\alpha_{i}}(n): n \geq 0\right\}$ at time $t=k+1, \ldots, k-1+p_{i}$ are given by the following lemma.

\section{Lemma 6}

$\forall t \in \mathbb{N}$ such that $1 \leq t \leq 3 m-1-\alpha_{i}$, we have $x^{\alpha_{i}}(k+t)=0$. 
It is easy to verify that $\forall i \in \mathbb{N}, 0 \leq i \leq-1+\rho(m)$, we have:

$$
\operatorname{PPos}\left(\alpha_{i}, j\right)=Q\left(\alpha_{i}, j\right) \forall j, \quad 1 \leq j \leq 3 m-1-\alpha_{i}
$$

\section{Lemma 7}

There exists $\bar{a}, \phi^{\alpha_{i}} \in \mathbb{R}^{k}$ and $\bar{\theta} \in \mathbb{R}$ such that :

$$
\operatorname{Per}\left(\bar{a}, \bar{\theta}, \phi^{\alpha_{i}}\right)=p_{i}
$$

Lemma $8 \forall t, i \in \mathbb{N}, t \geq k$ and $0 \leq i \leq-1+\rho(m)$

$$
\mu\left(m, \alpha_{i}\right) \leq \sum_{j=1}^{k} x^{\alpha_{i}}(t-j) \leq 1+\mu\left(m, \alpha_{i}\right) .
$$

In order to present some properties of the sequence $\left\{x^{\alpha_{i}}(n): n \geq 0\right\}$, we introduce the following notation:

Notation 1 Let us define $S 1\left(\alpha_{i}, n\right)$ as:

$$
S 1\left(\alpha_{i}, n\right)=\sum_{j=1}^{k} \bar{a}_{j} x^{\alpha_{i}}(n-j)
$$

and let $\lambda$ be a strictly negative real number such that: $\forall i, 0 \leq i \leq \rho(m)-1$

$$
\max \left\{S 1\left(\alpha_{i}, n\right)-\bar{\theta}: S 1\left(\alpha_{i}, n\right)<\bar{\theta} \text { and } n \geq k\right\} \leq \lambda
$$

Lemma $9 \forall i, n \in \mathbb{N}$ such that $0 \leq i \leq-1+\rho(m)$ and $n \geq k$,

$$
\begin{gathered}
S 1\left(\alpha_{i}, n\right) \in\left[-2\left(1+\mu\left(m, \alpha_{i}\right)\right), \bar{\theta}-1\right] \cup\{\bar{\theta}\}, \\
\lambda \in[-1,0[.
\end{gathered}
$$


Let $\left\{v^{\alpha_{i}}(n): n \geq 0\right\}$ be the sequence whose first $k$ terms are defined as follows:

$$
v^{\alpha_{i}}(0) v^{\alpha_{i}}(1) \ldots v^{\alpha_{i}}(k-1)=x^{\alpha_{i}}(1) \cdots x^{\alpha_{i}}(k-1) \overline{x^{\alpha_{i}}(k)},
$$

and the other terms are generated by the following neuronal recurrence equation:

$$
v^{\alpha_{i}}(n)=\mathbf{1}\left[\sum_{j=1}^{k} \bar{a}_{j} v^{\alpha_{i}}(n-j)-\bar{\theta}\right], \quad n \geq k .
$$

Remark 1 The term $x^{\alpha_{i}}(k)$ is equal to 1 , this implies that $v^{\alpha_{i}}(k-1)$ is equal to 0 .

The parameters $\bar{a}_{j}, 1 \leq j \leq k$ and $\bar{\theta}$ used in neuronal recurrence Equation (31) are those defined in Equations (17), (18) and (24).

The following lemma, which is easy to prove, characterizes the evolution of the sequence $\left\{v^{\alpha_{i}}(n): n \geq 0\right\}$.

Lemma 10 In the evolution of the sequence $\left\{v^{\alpha_{i}}(n): n \geq 0\right\}, \forall t \in \mathbb{N}, t \geq k$ we have:

(a) $v^{\alpha_{i}}(t)=0$,

(b) $\sum_{j=1}^{k} \bar{a}_{j} v^{\alpha_{i}}(t-j) \leq \bar{\theta}-2$,

(c) The sequence $\left\{v^{\alpha_{i}}(n): n \geq 0\right\}$ describes a transient of length $k-p_{i}$ and a fixed point.

The instability of the sequence $\left\{x^{\alpha_{i}}(n): n \geq 0\right\}$ occurs as a result of the convergence of the sequence $\left\{v^{\alpha_{i}}(n): n \geq 0\right\}$ to $00 \cdots 00$.

Notation 2. $h=\rho(m) \times k=(6 m-1) \times(\rho(m))^{2}$ is the length of the memory of some neuronal recurrence equations.

Let us also note:

$L_{0}(d)= \begin{cases}\rho(m) \times l c m\left(p_{d+1}, p_{d+2}, \ldots, p_{-2+\rho(m)}, p_{-1+\rho(m)}\right), & \text { if } 0 \leq d \leq-2+\rho(m) \\ 1, & \text { if } d=-1+\rho(m) .\end{cases}$ 


$$
\begin{aligned}
L_{1}(d) & =\rho(m) \times l c m\left(p_{0}, p_{1}, \ldots, p_{d}\right), 0 \leq d \leq \rho(m)-1 \\
L_{2} & =\rho(m) \times l c m\left(p_{0}, p_{1}, \ldots, p_{-1+\rho(m)}\right)
\end{aligned}
$$

$L_{0}(d), L_{1}(d)$ and $L_{2}$ represent the periods of some neuronal recurrence equations.

Let $\{y(n): n \geq 0\}$ be the sequence whose first $h$ terms are defined as follows:

$\forall j \in \mathbb{N}, 0 \leq j \leq k-1 \quad y((\rho(m) \times j)+i)=x^{\alpha_{i}}(1+j), \quad 0 \leq i \leq-1+\rho(m)$

and the other terms are generated by the following neuronal recurrence equation:

$$
y(n)=\mathbf{1}\left[\sum_{f=1}^{h} b_{f} y(n-f)-\theta_{1}\right] ; n \geq h
$$

where

$$
\begin{aligned}
& b_{f}= \begin{cases}\bar{a}_{j}, & \text { if } f=\rho(m) \times j, \quad 1 \leq j \leq k \\
0, & \text { otherwise. }\end{cases} \\
& \theta_{1}=\bar{\theta} .
\end{aligned}
$$

The parameters $\bar{a}_{j}$ are those defined in Equations (17) and (18). The parameters $\bar{\theta}$ and $k$ are defined in Equations (24) and (25).

Remark 2 (a) The first $h$ terms of the sequence $\{y(n): n \geq 0\}$ are obtained by shuffling the $k$ terms of each subsequence $\left\{x^{\alpha_{i}}(n): 1 \leq n \leq k\right\}$ where $0 \leq i \leq-1+\rho(m)$.

(b) The neuronal recurrence equation (36) is obtained by applying the construction of Lemma 1 to the neuronal recurrence Equation (16) whose parameters are given in Equations (17), (18), (24) and (25).

From the fact that the sequence $\{y(n): n \geq 0\}$ is the shuffle of the $\rho(m)$ subsequences and from its construction, we can write: 
Lemma $11 \forall t \in \mathbb{N}$ such that $t=q \times \rho(m)+i$ with $q \in \mathbb{N}$ and $0 \leq i \leq$ $-1+\rho(m)$, we have:

$$
y(t)=x^{\alpha_{i}}(1+q) .
$$

The next lemma gives the period of the sequences $\{y(n): n \geq 0\}$.

Lemma 12 The sequence $\{y(n): n \geq 0\}$ describes a cycle of length $L_{2}$.

$\forall d \in \mathbb{N}$ such that $0 \leq d \leq-1+\rho(m)$, we denote by $\{w(n, d): n \geq 0\}$ the sequence whose first $h$ terms are defined as:

$$
\forall i, 0 \leq i \leq d \quad w(\rho(m) j+i, d)= \begin{cases}x^{\alpha_{i}}(1+j), & 0 \leq j \leq k-2 \\ \overline{x^{\alpha_{i}}(k)}, & \mathrm{j}=\mathrm{k}-1 .\end{cases}
$$

and

$\forall i, d+1 \leq i \leq-1+\rho(m) w(\rho(m) j+i, d)=y\left(\rho(m) j+i+L_{1}(d)\right) ; 0 \leq j \leq k-1(40)$

The first $h$ terms of the sequence $\{w(n, d): n \geq 0\}$ are obtained by shuffling the $k$ terms of each of the sequences:

$$
v^{\alpha_{i}}(0) v^{\alpha_{i}}(1) v^{\alpha_{i}}(2) \ldots v^{\alpha_{i}}(k-1) ; 0 \leq i \leq d
$$

and

$x^{\alpha_{i}}\left(1+\gamma_{i}(d)\right) x^{\alpha_{i}}\left(2+\gamma_{i}(d)\right) x^{\alpha_{i}}\left(3+\gamma_{i}(d)\right) \ldots x^{\alpha_{i}}\left(k+\gamma_{i}(d)\right) ; d+1 \leq i \leq-1+\rho(m)$

where:

$$
\frac{L_{1}(d)}{\rho(m)} \equiv \gamma_{i}(d) \quad\left(\bmod p_{i}\right) ; d+1 \leq i \leq-1+\rho(m)
$$

The other terms of the sequence $\{w(n, d): n \geq 0\}$ are generated by the following neuronal recurrence equation:

$$
w(n, d)=\mathbf{1}\left[\sum_{f=1}^{h} b_{f} w(n-f, d)-\theta_{1}\right] ; n \geq h
$$

The next lemma gives the period of the sequence $\{w(n, d): n \geq 0\}$. 
Lemma 13 The sequence $\{w(n, d): n \geq 0\}$ generates a transient of length $\left(\rho(m) \times\left(k-p_{d}-1\right)\right)+d+1$ and a cycle of length $L_{0}(d)$.

Notation 3 Let us define $S 2(n), S 3(n, d)$ as:

$$
\begin{aligned}
S 2(n) & =\sum_{f=1}^{h} b_{f} y(n-f), \\
S 3(n, d) & =\sum_{f=1}^{h} b_{f} w(n-f, d) .
\end{aligned}
$$

Remark 3 On the basis of the composition of automata [5] and the definition of $\lambda$, we can conclude that:

- $\max \left\{S 2(n)-\theta_{1}: S 2(n)<\theta_{1}\right.$ and $\left.n \geq h\right\}=\max \{S 1(r, n)-\bar{\theta}:$ $S 1(r, n)<\bar{\theta}$ and $n \geq k\} \leq \lambda$, and

- $\max \left\{S 3(n, d)-\theta_{1}: S 3(n, d)<\theta_{1}\right.$ and $\left.n \geq h\right\}=\max \{S 1(r, n)-\bar{\theta}:$ $S 1(r, n)<\bar{\theta}$ and $n \geq k\} \leq \lambda$.

\subsection{Results on the dynamics of sequences $y$ and $w$}

In this subparagraph, we recall and give some interesting results on dynamics of the sequences $\{y(n): n \geq 0\}$ and $\{w(n, d): n \geq 0\}$.

The following lemma characterizes the sequence $\{y(n): n \geq 0\}$ and $\{w(n, d)$ : $n \geq 0\}$ in terms of the sum of $h$ consecutive terms.

Lemma $14 \forall n, d \in \mathbb{N}$, such that $n \geq h$ and $0 \leq d \leq \rho(m)-1$

- $\sum_{i=0}^{-1+\rho(m)} \mu\left(m, \alpha_{i}\right) \leq \sum_{f=1}^{h} y(n-f) \leq \rho(m)+\sum_{i=0}^{-1+\rho(m)} \mu\left(m, \alpha_{i}\right)$,

- $\sum_{i=d+1}^{-1+\rho(m)} \mu\left(m, \alpha_{i}\right) \leq \sum_{f=1}^{h} w(n-f, d) \leq-d-1+\rho(m)+\sum_{i=0}^{-1+\rho(m)} \mu\left(m, \alpha_{i}\right)$.

Definition 1 Let us define the terms of the sequence $\{\operatorname{tim}(i, l, q) \quad: l, q \in$ $\mathbb{N}$ and $0 \leq i \leq-1+\rho(m)\}$ as:

$$
\operatorname{tim}(i, l, q)=(l \times \rho(m))+i+q: \quad l, q \in \mathbb{N} \text { and } 0 \leq i \leq-1+\rho(m) .
$$


From Lemma 11] one can easily deduce that:

$$
\begin{aligned}
& y(\operatorname{tim}(i, l, 0)) y(\operatorname{tim}(i, l+1,0)) \ldots y(\operatorname{tim}(i, l+k-1,0))= \\
& x^{\alpha_{i}}(1+l) \ldots x^{\alpha_{i}}(l+k) ; 0 \leq i \leq-1+\rho(m) .
\end{aligned}
$$

From Lemma 11 or Equation (45), we also easily deduce that the terms of the sequence $\{y(n): n \geq 0\}$ verify the following relation:

$$
\begin{gathered}
y\left(\operatorname{tim}\left(i, 0,-\rho(m)+L_{1}(d)\right)\right) y\left(\operatorname{tim}\left(i, 1,-\rho(m)+L_{1}(d)\right)\right) \ldots y\left(\operatorname{tim}\left(i, k-1,-\rho(m)+L_{1}(d)\right)\right)= \\
\underbrace{00 \ldots 0}_{\beta\left(m, \alpha_{i}\right)} \underbrace{10 \ldots 0}_{p_{i}} \ldots \underbrace{10 \ldots 00}_{p_{i}} ; 0 \leq i \leq d \\
y\left(\operatorname{tim}\left(i, 0,-\rho(m)+\alpha_{i}\right) \times p_{i}\right.
\end{gathered}
$$

For all $d \in \mathbb{N}$ such that $0 \leq d \leq-1+\rho(m)$, we note $B_{0}(d)$ the set of integers $f$ which verify Equations (47) and (48):

$$
\begin{gathered}
1 \leq f \leq h-d \\
y\left(-f-\rho(m)+h+L_{1}(d)\right)=1 .
\end{gathered}
$$

Comment : By considering the following terms: $y\left(-\rho(m)+L_{1}(d)\right) y(1-$ $\left.\rho(m)+L_{1}(d)\right) \ldots y\left(-\rho(m)+L_{1}(d)+h-1\right)$, it is possible by easy computation to build the set $B_{0}(d)$. In a bid to give the algebraic expression of the set $B_{0}(d)$, let us define the set $C 0\left(i, p_{i}\right)$ as follows:

$C 0\left(i, p_{i}\right)=\left\{-i+\rho(m)+\left(\rho(m) \times p_{i} \times j\right): j=0,1, \ldots, \mu\left(m, \alpha_{i}\right)\right\}, 0 \leq i \leq \rho(m)-1$. 
From the definition of the terms $y(0) y(1) \ldots y(h-1)$ (see Equation (35)), it is easy to see that: $\bigcup_{i=0}^{\rho(m)-1} C 0\left(i, p_{i}\right)$ represents the set of indices $j$ such that $y(h-j)=1$.

Let us define the set $C 1(n, y)$ as follows:

$$
C 1(n, y)=\{j: y(h+n-j)=1\}
$$

It is easy to see that:

$$
\begin{aligned}
\ell \in \bigcup_{i=0}^{\rho(m)-1} C 0\left(i, p_{i}\right) & \Longleftrightarrow y(h-\ell)=1 \text { and } 1 \leq \ell \leq h, \\
& \Longleftrightarrow y(h+n-(\ell+n))=1 \text { and } 1 \leq \ell \leq h, \\
& \Longleftrightarrow \ell+n \in C 1(n, y) \text { and } 1 \leq \ell \leq h .
\end{aligned}
$$

Consequently:

$$
\ell \in \bigcup_{i=0}^{\rho(m)-1} C 0\left(i, p_{i}\right) \Longleftrightarrow \ell+n \in C 1(n, y) \text { and } 1 \leq \ell \leq h,
$$

Based on the period of the subsequence $\left\{x^{\alpha_{i}}(n): n \geq 0\right\}$ and on the fact that $y$ is a shuffle of the subsequences $x^{\alpha_{i}}(0 \leq i \leq \rho(m)-1)$, we deduce that:

$$
\left.\begin{array}{l}
\ell+n \in C 1(n, y), \\
\ell \in C 0\left(i, p_{i}\right),
\end{array}\right\} \Longrightarrow \ell+n-\left(\rho(m) \times p_{i}\right) \in C 1(n, y) .
$$

Let us denote $C 2(i, d)$ the following set:

$$
C 2(i, d)=\left\{j:-i+\rho(m)+L_{1}(d)-\rho(m) \equiv j \quad\left(\bmod \rho(m) \times p_{i}\right)\right\} .
$$

The set $C 2(i, d)$ contains the only element $j\left(0 \leq j<\rho(m) \times p_{i}\right)$ such that $-i+\rho(m)+L_{1}(d)-\rho(m) \equiv j\left(\bmod \rho(m) \times p_{i}\right)$. By using the Equation (51) and the fact that $-i+\rho(m) \in C 0\left(i, p_{i}\right)$, we deduce the following implications:

$$
\left.\begin{array}{l}
-i+\rho(m) \in C 0\left(i, p_{i}\right), \\
j \in C 2(i, d), \\
j \neq 0,
\end{array}\right\} \Longrightarrow j \in C 1(n, y)
$$




$$
\left.\begin{array}{l}
-i+\rho(m) \in C 0\left(i, p_{i}\right), \\
j \in C 2(i, d), \\
j=0,
\end{array}\right\} \Longrightarrow \rho(m) \times p_{i} \in C 1(n, y)
$$

We build the set $C 3(i, d)$ as follows:

$$
C 3(i, d)=\left\{\ell: \ell \equiv j \quad\left(\bmod \rho(m) \times p_{i}\right), 1 \leq \ell \leq h, \ell \in C 2(i, d)\right\} .
$$

It is easy to see that:

$$
B_{0}(d)=\bigcup_{i=0}^{\rho(m)-1} C 3(i, d)
$$

Let us denote $A(d)$ the following set:

$$
\begin{aligned}
A(d) & =\bigcup_{i=0}^{d} B_{i}(d) \text { where } \\
B_{i+1}(d) & =\left\{1+\jmath: \jmath \in B_{i}(d)\right\}
\end{aligned}
$$

Based on:

- the evolution of the neuronal recurrence equation $y$,

- on the definition of the set $B_{i}(d)$,

it is easy to verify that:

$\forall \ell \in \mathbb{N}, 0 \leq \ell \leq d$ and $\forall f \in B_{\ell}(d)$ we have $y\left(-f-\rho(m)+h+\ell+L_{1}(d)\right)=1$.

\section{Construction of the neuronal recurrence equa- tion $z$}

The basic idea is to construct a sequence $\{z(n, d): n \geq 0\}$ whose terms are generated by the following neuronal recurrence equation:

$$
z(n, d)=\mathbf{1}\left[\sum_{f=1}^{h} c(f, d) z(n-f, d)-\theta_{2}(d)\right],
$$


and whose first $h$ terms are initialized as follows:

$$
z(f, d)=y(f) \quad ; \quad 0 \leq f \leq h-1
$$

which exploits the instability of the sequences $\left\{x^{\alpha_{i}}(n): n \geq 0\right\}$ to converge to the sequence $\{w(n, d): n \geq 0\}$.

We define the parameters $c(f, d)$ and $\theta_{2}(d)$ as follows:

$$
\begin{gathered}
c(f, d)= \begin{cases}b_{f} & 1 \leq f \leq h \text { and } f \notin A(d), \\
b_{f}+\beta(d) & 1 \leq f \leq h \text { and } f \in A(d) .\end{cases} \\
\theta_{2}(d)=\theta_{1}+\xi(d),
\end{gathered}
$$

where:

$$
\begin{aligned}
\beta(d) & =\frac{\lambda}{\operatorname{Tot}(d)}, \\
\xi(d) & =\lambda-\frac{\beta(d)}{8}, \\
\operatorname{Tot}(d) & =\operatorname{card} B_{\ell}(d), 0 \leq \ell \leq d .
\end{aligned}
$$

We have defined the parameters $c(f, d)$ so that the sequence $\{z(n, d): n \geq 0\}$ converges to the sequence $\{w(n, d): n \geq 0\}$.

Remark 4 The terms of the sequence $\{z(n, d): n \geq 0\}$ verify the following relation:

$$
\begin{aligned}
& z(\operatorname{tim}(i, 0,0), d) z(\operatorname{tim}(i, 1,0), d) \ldots z(\operatorname{tim}(i, k-1,0), d)= \\
& x^{\alpha_{i}}(1) x^{\alpha_{i}}(2) \ldots x^{\alpha_{i}}(k-1) x^{\alpha_{i}}(k) ; 0 \leq i \leq-1+\rho(m) .
\end{aligned}
$$

Notation 4 Let $Q 2(n, d)$ be defined as follows:

$$
Q 2(n, d)=\beta(d) \sum_{f \in A(d)} z(n-f, d)-\xi(d) .
$$


We establish below a proposition which states a relation between $z(n, d), S 2(n)$ and $Q 2(n, d)$.

Proposition 3 If

$z(n-i, d)=y(n-i)$ for all $i$ such that $1 \leq i \leq h$ and $n-i \equiv 0 \quad(\bmod \rho(m))$

Then

$$
z(n, d)=\mathbf{1}\left[S 2(n)+Q 2(n, d)-\theta_{1}\right] .
$$

Below, we establish two propositions which provide the link between the value of $Q 2(n, d)$ and the sequence $z(n, d)$.

\section{Proposition 4 If}

$$
\sum_{f \in A(d)} z(n-f, d)=\operatorname{Tot}(d)
$$

Then

$$
Q 2(n, d)=\frac{\beta(d)}{8} .
$$

Proposition 5 If

$$
\sum_{f \in A(d)} z(n-f, d) \leq \operatorname{Tot}(d)-1
$$

Then

$$
\frac{-7 \beta(d)}{8} \leq Q 2(n, d) \leq-\xi(d) .
$$

In the next two lemmas, we show the relations between $B_{0}(d), A(d)$ and $\operatorname{Tot}(d)$.

Lemma $15 \forall d, n \in \mathbb{N}$ such that $0 \leq d \leq \rho(m)-1$

If

$$
n \not \equiv h-\rho(m)+L_{1}(d) \quad\left(\bmod L_{2}\right)
$$

Then

$$
\sum_{f \in B_{0}(d)} y(n-f)<\operatorname{Tot}(d)
$$


Lemma $16 \forall n \in \mathbb{N}$ such that $n \equiv \ell\left(\bmod L_{2}\right)$

If

$$
\ell \notin\left\{-\rho(m)+h+L_{1}(d), 1-\rho(m)+h+L_{1}(d), \ldots, d-\rho(m)+h+L_{1}(d)\right\}
$$

Then

$$
\sum_{f \in A(d)} y(n-f)<\operatorname{Tot}(d)
$$

We want to exploit the fact that:

- $\sum_{f \in A(d)} z(n-f, d)=\operatorname{Tot}(d)$ implies that $Q 2(n, d)=\frac{\beta(d)}{8}<0$.

- $\sum_{f \in A(d)} z(n-f, d) \leq \operatorname{Tot}(d)-1$ implies that $\frac{-7 \beta(d)}{8} \leq Q 2(n, d) \leq-\xi(d)$.

- $\forall t, 0 \leq t \leq \rho(m)-1$ we have $\sum_{f=1}^{h} b_{f} y\left(h-\rho(m)+t-f+L_{1}(-1+\rho(m))\right)=$ $\bar{\theta}$.

- the instability of the sequence $\left\{x^{\alpha_{i}}(n): n \geq 0\right\}$.

to prove that the sequence $\{z(n, d): n \geq 0\}$ converges to the sequence $\{w(n, d)$ : $n \geq 0\}$. We intend to divide the dynamic of the sequence $\{z(n, d): n \geq 0\}$ into five phases.

\section{Dynamical behavior of the neuronal recurrence equation $z$}

This study is done in two steps : first we analyse the transient phase and next the cyclic phase. Subsequently, we suppose that $d$ verifies the following equation

$$
d \in \mathbb{N} \text { and } 0 \leq d \leq \rho(m)-1
$$




\subsection{Transient Phase}

The transient phase of the sequence $\{z(n, d): n \geq 0\}$ unfolds during four phases.

Phase 1. We want that the dynamics of the sequence $\{z(n, d): n \geq 0\}$ verifies the relation: $\forall t \in \mathbb{N}$ such that $0 \leq t \leq L_{1}(d)+h-1-\rho(m)$, we have:

$$
z(t, d)=y(t)
$$

Phase 1 starts at time 0 and finishes at time $L_{1}(d)+h-1-\rho(m)$.

In the lemma below, we prove that the sequence $\{z(n, d): n \geq 0\}$ verifies the properties of phase 1.

Lemma 17 In the evolution of the neuronal recurrence equation $\{z(n, d): n \geq$ $0\}, \forall t \in \mathbb{N}$ such that $0 \leq t \leq L_{1}(d)+h-1-\rho(m)$, we have:

$$
z(t, d)=y(t)
$$

Phase 2. We want that $\forall t \in \mathbb{N}$ such that $L_{1}(d)+h-\rho(m) \leq t \leq$ $L_{1}(d)+h-\rho(m)+d$, we have:

$$
z(t, d)=0 \text { even when } y(t)=1 .
$$

Phase 2 occurs from time $h-\rho(m)+L_{1}(d)$ to time $L_{1}(d)+h-\rho(m)+d$. In the Lemma 18, we prove that the sequence $\{z(n, d): n \geq 0\}$ verifies the properties of phase 2 .

Lemma $18 \forall t \in \mathbb{N}$ such that $L_{1}(d)+h-\rho(m) \leq t \leq L_{1}(d)+h-\rho(m)+d$, we have:

$$
z(t, d)=0 \text { even when } y(t)=1 \text {. }
$$

After phase 2, the behavior of the sequence $\{z(n, d): n \geq 0\}$ begins to be different from the behavior of the sequence $\{y(n): n \geq 0\}$. After phase 2 , the sequence $\{z(n, d): n \geq 0\}$ begins its convergence to the sequence $\{w(n, d): n \geq$ $0\}$.

Phase 3. This phase starts at time $L_{1}(d)+h-\rho(m)+d+1$, and finishes at time $L_{1}(d)+h-1$. 
Lemma 19 In the evolution of the neuronal recurrence equation $\{z(n, d): n \geq$ $0\}, \forall t \in \mathbb{N}$ such that $L_{1}(d)+h-\rho(m)+d+1 \leq t \leq L_{1}(d)+h-1$ we have:

$$
z(t, d)=y(t)
$$

Remark 5 Based on the Lemma[11, Lemma 17, Lemma[18 and Lemma 19, we easily deduce that the terms of the sequence $\{z(n, d): n \geq 0\}$ verify the following relations:

$$
\begin{gathered}
z\left(\operatorname{tim}\left(i, 0, L_{1}(d)\right), d\right) z\left(\operatorname{tim}\left(i, 1, L_{1}(d)\right), d\right) \ldots z\left(\operatorname{tim}\left(i, k-1, L_{1}(d)\right), d\right)= \\
v^{\alpha_{i}}(0) v^{\alpha_{i}}(1) \ldots v^{\alpha_{i}}(k-1), 0 \leq i \leq d \\
z\left(\operatorname{tim}\left(i, 0, L_{1}(d)\right), d\right) z\left(\operatorname{tim}\left(i, 1, L_{1}(d)\right), d\right) \ldots z\left(\operatorname{tim}\left(i, k-1, L_{1}(d)\right), d\right)= \\
x^{\alpha_{i}}\left(1+\gamma_{i}(d)\right) x^{\alpha_{i}}\left(2+\gamma_{i}(d)\right) \ldots x^{\alpha_{i}}\left(k+\gamma_{i}(d)\right), d+1 \leq i \leq-1+\rho(m)
\end{gathered}
$$

where $\gamma_{i}(d)$ is defined in Equation [43).

\section{Remark 6 If}

$$
d=\rho(m)-1
$$

Then it is clear that the phase 3 doesn't exists.

Phase 4. This phase starts at time $L_{1}(d)+h$ and finishes at time $L_{1}(d)+$ $h+d+\left(\rho(m) \times\left(k-1-p_{d}\right)\right)$. This phase corresponds to the transient phase of the neuronal recurrence equation $\{w(n, d): n \geq 0\}$.

Lemma 20 In the evolution of the neuronal recurrence equation $\{z(n, d): n \geq$ $0\}, \forall t \in \mathbb{N}$ such that $0 \leq t \leq\left(\rho(m) \times\left(k-1-p_{d}\right)\right)+d$, we have:

$$
z\left(L_{1}(d)+h+t, d\right)=w(h+t, d) .
$$

Notation 5 We set $L_{3}(d)=L_{1}(d)+2 h+d-\left(\rho(m) \times\left(1+p_{d}\right)\right)$, which represents the end of the fourth phase and we set $L_{4}(d)=L_{3}(d)-h+1$ which represents the beginning of the cyclic phase. 
Remark 7 Based on the Lemma 17, Lemma 18, Lemma 19, and Lemma [20, we easily deduce that the terms of the sequence $\{z(n, d): n \geq 0\}$ verify the following relations:

$$
\begin{gathered}
z\left(\operatorname{tim}\left(i, 0, L_{4}(d)\right), d\right) z\left(\operatorname{tim}\left(i, 1, L_{4}(d)\right), d\right) \ldots z\left(\operatorname{tim}\left(i, k-1, L_{4}(d)\right), d\right)= \\
x^{\alpha_{i+d+1}}\left(\gamma_{i+d+1}(d)+k-p_{d}\right) x^{\alpha_{i+d+1}}\left(1+\gamma_{i+d+1}(d)+k-p_{d}\right) \ldots \\
x^{\alpha_{i+d+1}}\left(2 k-1+\gamma_{i+d+1}(d)-p_{d}\right) ; 0 \leq i \leq \rho(m)-d-2 \\
z\left(\operatorname{tim}\left(i, 0, L_{4}(d)\right), d\right) z\left(\operatorname{tim}\left(i, 1, L_{4}(d)\right), d\right) \ldots z\left(\operatorname{tim}\left(i, k-1, L_{4}(d)\right), d\right)= \\
\underbrace{000 \ldots 000}_{k} ; \rho(m)-d-1 \leq i \leq \rho(m)-1 .
\end{gathered}
$$

The sequence $\{z(n, d): n \geq 0\}$ describes a cycle during its fifth step. The subparagraph below is devoted to this study.

\subsection{Cyclic Phase}

Phase 5. This phase starts at time $L_{4}(d)$ and describes a cycle of length $L_{0}(d)$.

\section{Lemma 21}

$z\left(t+L_{4}(d), d\right)=w\left(t+h+d+1+\left(\rho(m) \times\left(-1-p_{d}\right)\right), d\right) ; \forall t \in \mathbf{N}$.

We have shown the following Lemma:

Lemma 22 The sequence $\{z(n, d): n \geq 0\}$ describes a transient of length $L_{4}(d)$ and a cycle of length $L_{0}(d)$.

It is easy to see that:

- we can build by perturbation the neuronal recurrence equation $\{z(n, 0)$ : $n \geq 0\}$ from the neuronal recurrence equation $\{y(n): n \geq 0\}$

- we can build by perturbation the neuronal recurrence equation $\{z(n, d+$ 1) $: n \geq 0\}$ from the neuronal recurrence equation $\{z(n, d): n \geq 0\}$ 
This second item is obtained by the following transformations:

$$
c(f, 1+d)= \begin{cases}c(f, d), & \text { if } f \notin A(d) \cup A(1+d) \\ c(f, d)-\beta(d)+\beta(1+d), & \text { if } f \in A(d) \cap A(1+d) \\ c(f, d)-\beta(d), & \text { if } f \in A(d) \cap \overline{A(1+d)} \\ c(f, d)+\beta(1+d), & \text { if } f \in \overline{A(d)} \cap A(1+d) \\ \theta_{2}(d+1)=\theta_{2}(d)-\xi(d)+\xi(d+1) .\end{cases}
$$

The main result of the paper is:

Theorem $1 \forall m, d \in \mathbb{N}$ such that $\rho(m) \geq 2$ and $0 \leq d \leq-1+\rho(m)$. We construct a set of $\rho(m)+1$ neuronal recurrence equations which verify:

- the neuronal recurrence equation $\{y(n): n \geq 0\}$ describes a cycle of length $L_{1}(-1+\rho(m))$

- by perturbation, we can build the neuronal recurrence equation $\{z(n, 0)$ : $n \geq 0\}$ from the neuronal recurrence equation $\{y(n): n \geq 0\}$. The period of the neuronal recurrence equation $\{z(n, 0): n \geq 0\}$ is a divisor of the period of the neuronal recurrence equation $\{y(n): n \geq 0\}$

- by perturbation, we can build the neuronal recurrence equation $\{z(n, d+1)$ : $n \geq 0\}$ from the neuronal recurrence equation $\{z(n, d): n \geq 0\}$. The period of the neuronal recurrence equation $\{z(n, d+1): n \geq 0\}$ is a divisor of the period of the neuronal recurrence equation $\{z(n, d): n \geq 0\}$

- the period of the neuronal recurrence equation $\{z(n,-1+\rho(m)): n \geq 0\}$ is 1 (i.e. a fixed point).

Remark 8 The new contribution in this paper with respect to the previous work [13] is that: 
- in the paper [13], the sequence $\{z(n): n \geq 0\}$ is a composition of the $s+1$ subsequences of periods $p_{0}, p_{1}, \ldots, p_{s}$ and $3 m-1$, in the evolution of the sequence $\{z(n): n \geq 0\}$, the subsequence of period $3 m-1$ vanishes and converges to the null sequence $000 \ldots 000 \ldots$ This fact appears in the formula of transient length and in the formula of the cycle length of the sequence $\{z(n): n \geq 0\}$ which are respectively $(s+1)(3 m+1+$ $\left.\operatorname{lcm}\left(p_{0}, p_{1}, \ldots, p_{s-1}, 3 m-1\right)\right)$ and $(s+1) l c m\left(p_{0}, p_{1}, \ldots, p_{s-1}, 3 m-1\right)$. It is clear that in this case, the periods $p_{0}, p_{1}, \ldots, p_{s-1}$ of the subsequences intervene in the formula of transient and in the formula of period.

- in the following paper, the sequence $\{z(n, d): n \geq 0\}$ is a composition of the $\rho(m)$ subsequences of periods $p_{0}, p_{1}, \ldots, p_{-1+\rho(m)}$ and in the evolution of the sequence $\{z(n, d): n \geq 0\}$, the subsequences of period $p_{0}, p_{1}, \ldots, p_{d}$ vanish and converge to the null sequence $000 \ldots 000 \ldots$ This fact appears in the formula of transient length and on the cycle length of the sequence $\{z(n, d): n \geq 0\}$ which are respectively $\rho(m) \times$ $l c m\left(p_{0}, p_{1}, \ldots, p_{d}\right)+h+d+1-\left(\rho(m) \times\left(1+p_{d}\right)\right), 0 \leq d \leq-1+\rho(m)$ and $\rho(m) \times \operatorname{lcm}\left(p_{d+1}, p_{d+2}, \ldots, p_{-1+\rho(m)}\right)$ if $0 \leq d \leq-2+\rho(m)$ and 1 if $d=\rho(m)-1$. It is clear that in this case, there doesn't exists a period $p_{i}$ of a subsequence which intervenes in the formulas of transient and in the formula of period.

- the difference mentioned in the expressions of the formula of transient and in the formula of period on the following paper and of the paper [13] imply that the concept used in the construction of the set A ( see page 520 of the paper [13] ) is fondamentally different from the concept used in the construction of the set $A(d)$ (see Equation (54)).

- in the paper [13], we build only one neuronal recurrence equation $\{z(n)$ : $n \geq 0\}$ whereas on the following paper we build $\rho(m)$ neuronal recurrence equation $\{z(n, d): n \geq 0\}, 0 \leq d \leq \rho(m)-1$. 
Let us note $e$ an integer such that:

$$
\forall i \in \mathbb{N}, 0 \leq i \leq \rho(m)-1 \text { we have } \beta\left(m, \alpha_{e}\right) \leq \beta\left(m, \alpha_{i}\right)
$$

Subsequently, we suppose that:

$$
d<\beta\left(m, \alpha_{e}\right) .
$$

Let us note $\widetilde{z}(0, d) \widetilde{z}(1, d) \ldots \widetilde{z}(h-1, d)$ the following $h$ terms:

$$
\begin{aligned}
& \widetilde{z}(i, d)=y(i) \text { for } i \text { such that } \beta\left(m, \alpha_{e}\right)-d \leq i \leq h-1 \\
& \widetilde{z}(i, d) \in\{0,1\} \text { for } i \text { such that } 0 \leq i \leq \beta\left(m, \alpha_{e}\right)-d-1
\end{aligned}
$$

The following lemma characterizes the basin of attraction of the sequence $\{z(n, d)$ : $n \geq 0\}$.

Lemma 23 If $d<\beta\left(m, \alpha_{e}\right)$ Then from the following initial configurations:

$$
\begin{aligned}
& y(0) y(1) \ldots y(h-1), \\
& \widetilde{z}(0, d) \widetilde{z}(1, d) \ldots \widetilde{z}(h-1, d)
\end{aligned}
$$

the neuronal recurrence equation $\{z(n, d): n \geq 0\}$ converges to the same basin of attraction.

\section{Conclusion}

We improve the fundamental lemma of composition of neuronal recurrence equations. From a neuronal recurrence equation that describes a cycle of length $L_{2}$, we construct a set of $\rho(m)$ neuronal recurrence equations $\{z(n, d): n \geq 0\}$ whose dynamics describe respectively the cycle of length $L_{0}(d)$ where $L_{0}(d)$ are the divisors of $L_{2}$. The neuronal recurrence equation $\{z(n,-1+\rho(m)): n \geq 0\}$ describes an exponential transient and a fixed point. By perturbation, we have

built the neuronal recurrence equation $\{z(n, d+1): n \geq 0\}$ from the neuronal 
recurrence equation $\{z(n, d): n \geq 0\}$ such that the period of the neuronal recurrence equation $\{z(n, d+1): n \geq 0\}$ is a divisor of the period of the neuronal recurrence equation $\{z(n, d): n \geq 0\}$. Thus, we have built a period-halving bifurcation of a neuronal recurrence equation. This result is inscribed in the framework of results on convergence time of neural networks [8, 16, 17]. The exponential convergence time of neuronal recurrence equations can be useful when we want to use it in cryptographic toolbox ( e.g. remote authentication, generation of pseudo-random numbers, ... ).

\section{Acknowledgements}

The author express his gratitude to Professor Michael Y. Li ( University of Alberta ) for useful discussions. The author also express his gratitude to the reviewer for useful remarks. This work was supported by LIRIMA , University of Yaounde 1, and by The Abdus Salam International Centre for Theoretical Physics (ICTP), Trieste, Italy.

\section{Appendix}

\section{Proof of Lemma 3}

The proof is subdivided into two cases:

First case: $\exists j, 1 \leq j \leq r$ such that $p_{j} \geq 2$

This case corresponds to Lemma 1 and it was proved by Cosnard, Tchuente and Tindo [5].

Second case: $\forall j, 1 \leq j \leq r$ we have $p_{j}=1$.

Without loss of generality, we suppose that the transient length of each sequence is 0 . It is easy to see that:

$$
p_{j}=1 \Longrightarrow x^{j}(n)=x^{j}(0), \forall n \in \mathbb{N} .
$$


It follows that $\mathrm{Per}$ is the period of the following sequence:

$$
\begin{aligned}
& x^{1}(0) x^{2}(0) \ldots x^{r}(0) x^{1}(1) x^{2}(1) \ldots x^{r}(1) \ldots x^{1}(\ell) x^{2}(\ell) \ldots x^{r}(\ell) \ldots= \\
& x^{1}(0) x^{2}(0) \ldots x^{r}(0) x^{1}(0) x^{2}(0) \ldots x^{r}(0) \cdots x^{1}(0) x^{2}(0) \ldots x^{r}(0) \ldots
\end{aligned}
$$

It is clear that $r$ is a multiple of the period, i.e. Per is a divisor of $r$.

\section{Proof of Lemma 4}

Without loss of generality, we can assume that for all $\jmath, 1 \leq \jmath<g$, we have $T_{\jmath} \leq T_{g}$. It suffices to apply the construction used in the demonstration of Lemma 1 to the sequences $\left\{x^{\jmath}(n): n \geq 0\right\}, 1 \leq \jmath \leq g$.

\section{Proof of Proposition 1}

The proof is given in [14] (see Proposition 1 of [14]).

\section{Proof of Proposition 2}

The proof is subdivided into two cases:

First case: $\rho(m)$ is even

$$
\begin{aligned}
\sum_{j \in \operatorname{Pos}\left(\alpha_{i}\right)} \bar{a}_{j} & =\sum_{\ell=1}^{2 \times \rho(m)} \bar{a}_{\ell \times p_{i}} \\
& =\sum_{\ell=1}^{(3 \times \rho(m)) / 2} 2-\sum_{\ell=1+((3 \times \rho(m)) / 2)}^{2 \times \rho(m)} 2 \\
& =2 \times \rho(m)
\end{aligned}
$$

Second case $: \rho(m)$ is odd and $\rho(m) \geq 3$ 


$$
\begin{aligned}
\sum_{j \in \operatorname{Pos}\left(\alpha_{i}\right)} \bar{a}_{j} & =\sum_{\ell=1}^{2 \times \rho(m)} \bar{a}_{\ell \times p_{i}} \\
& =\sum_{\ell=1}^{((3 \times \rho(m))-1) / 2} 2-\sum_{\ell=((3 \times \rho(m))+1) / 2}^{(2 \times \rho(m))-2} 2-\sum_{\ell=(2 \times \rho(m))-1}^{2 \times \rho(m)} 1 \\
& =2 \times \rho(m)
\end{aligned}
$$

\section{Proof of Lemma 5}

We have:

$$
\begin{aligned}
\sum_{j=1}^{k} \bar{a}_{j} x^{\alpha_{i}}(k-j) & =\sum_{j \in \operatorname{Pos}\left(\alpha_{i}\right)} \bar{a}_{j} x^{\alpha_{i}}(k-j)+\sum_{j \in D \backslash \operatorname{Pos}\left(\alpha_{i}\right)} \bar{a}_{j} x^{\alpha_{i}}(k-j) \\
& =\sum_{j \in \operatorname{Pos}\left(\alpha_{i}\right)} \bar{a}_{j} x^{\alpha_{i}}(k-j) \text { by application of the Equation(27) } \\
& =\sum_{j \in \operatorname{Pos}\left(\alpha_{i}\right)} \bar{a}_{j} \quad \text { by application of the Equation(26) } \\
& =2 \times \rho(m) \text { by application of the Proposition 2 }
\end{aligned}
$$

It follows that:

$$
\begin{aligned}
x^{\alpha_{i}}(k) & =\mathbf{1}\left(\sum_{j=1}^{k} \bar{a}_{j} x^{\alpha_{i}}(k-j)-\bar{\theta}\right) \\
& =\mathbf{1}(\bar{\theta}-\bar{\theta}) \quad \text { by application of the Equation (75) } \\
& =1
\end{aligned}
$$

\section{Proof of Lemma 6}

The result follows from the definition of $E\left(\alpha_{i}, d\right)$ and by application of Proposition 1 . 


\section{Proof of Lemma 7}

By application of Lemma 5 and Lemma 6 we deduce the result.

\section{Proof of Lemma 8}

From Equation (14) and Equation (15) we deduce the result.

\section{Proof of Lemma 9}

From Lemma 8, we have $\sum_{j=1}^{k} x^{\alpha_{i}}(n-j) \leq 1+\mu\left(m, \alpha_{i}\right)$, therefore it follows that $-2\left(1+\mu\left(m, \alpha_{i}\right)\right) \leq S 1\left(\alpha_{i}, n\right)$ because $-2 \leq \bar{a}_{j}$ for $j$ such that $1 \leq j \leq k$. Since $\bar{a}_{j}$ is an integer, on the basis of Equation (11), Equation (17) and Equation (18), we easily deduce that

$$
S 1\left(\alpha_{i}, n\right)<\bar{\theta} \Longrightarrow S 1\left(\alpha_{i}, n\right) \leq \bar{\theta}-1
$$

From the evolution of the sequence $\left\{x^{\alpha_{i}}(n): n \geq 0\right\}$, we know that for $t \in$ $\left\{k, k+1, \ldots, k+3 m-\alpha_{i}-2, k+3 m-\alpha_{i}-1\right\}$ the only $t$ at which $x^{\alpha_{i}}(t)=1$ is $k$ and that $S 1\left(\alpha_{i}, k\right)=\bar{\theta}$. From the fact that the sequence $\left\{x^{\alpha_{i}}(n): n \geq 0\right\}$ has period $3 m-\alpha_{i}$, we deduce that

$$
S 1\left(\alpha_{i}, n\right) \geq \bar{\theta} \Longrightarrow S 1\left(\alpha_{i}, n\right)=\bar{\theta}
$$

From the fact that $\forall n \in \mathbb{N}$,

$$
S 1\left(\alpha_{i}, n\right) \in\left[-2\left(1+\mu\left(m, \alpha_{i}\right)\right), \bar{\theta}-1\right] \cup\{\bar{\theta}\}
$$

It follows from the definition of $\lambda$ that $\lambda \in[-1,0[$. 


\section{Proof of Lemma 10}

From Equation (30), we have:

$$
v^{\alpha_{i}}(k-1)=\overline{x^{\alpha_{i}}(k)}=0 .
$$

From Equations (14), (15) and (30), we easily deduce that the structure of the initial terms of the sequence $\left\{v^{\alpha_{i}}(n): n \geq 0\right\}$ have the following form:

$$
v^{\alpha_{i}}(0) v^{\alpha_{i}}(1) \ldots v^{\alpha_{i}}(k-1)=\underbrace{0 \ldots 0}_{-1+\beta\left(m, \alpha_{i}\right)} \underbrace{10 \ldots 0}_{\mu\left(m, \alpha_{i}\right) p_{i}} \cdots \underbrace{10 \ldots 0}_{p_{i}} 0
$$

We want to prove by recurrence the part (a) of the Lemma:

Basis Case: $t=k$

Based on Equations (17), (18) and (76), we easily deduce that:

$$
\sum_{j=1}^{k} \bar{a}_{j} v^{\alpha_{i}}(k-j) \leq \bar{\theta}-2 .
$$

This implies that $v^{\alpha_{i}}(k)=0$.

Recurrence Hypothesis: we suppose that the property is true at all steps $\ell-k, \ell-k+1, \cdots, \ell-1$, i.e.

$$
\forall t \in \mathbb{N} \text { such that } k \leq t \leq \ell-1, \text { we have } v^{\alpha_{i}}(t)=0
$$

and

$\forall t \in \mathbb{N}$ such that $: t \leq k-1, v^{\alpha_{i}}(t)$ is defined by Equation (76).

\section{Step $\ell$ :}

Based on Equation (78), we easily deduce that:

$$
\sum_{j=1}^{k} \bar{a}_{j} v^{\alpha_{i}}(\ell-j) \leq \bar{\theta}-2 .
$$

This implies that $v^{\alpha_{i}}(\ell)=0$. We have shown the part (a) of the Lemma. The proof of part (b) is easily deduced from Equations (77) and (79). The part (c) is easily deduced from the part (a). 


\section{Proof of Lemma 12}

By applying the Lemma 1 and given the fact that the subsequences $\left\{x^{\alpha_{i}}(n)\right.$ : $n \geq 0\}$ describe a cycle of length $p_{i}$, we deduce the result.

\section{Proof of Lemma 13}

By application of Lemma 4 and given the fact that:

- the subsequence $\left\{v^{\alpha_{i}}(n): n \geq 0\right\}$ describes a transient of length $k-p_{i}$ and the following fixed point $\underbrace{000 \ldots 0000}_{k}$,

- $p_{-1+\rho(m)}<\ldots<p_{i+1}<p_{i}<\cdots<p_{1}<p_{0}$

- the subsequence $\left\{x^{\alpha_{i}}(n): n \geq 0\right\}$ describes a cycle of length $p_{i}$,

we deduce the result.

\section{Proof of Lemma 14}

Based on the fact that the sequence $\{y(n): n \geq 0\}$ is the shuffle of $\rho(m)$ subsequences of the form $\left\{x^{\alpha_{i}}(n): n \geq 0\right\}$ and on the Lemma 8 , we deduce that:

$$
\sum_{i=0}^{-1+\rho(m)} \mu\left(m, \alpha_{i}\right) \leq \sum_{f=1}^{h} y(n-f) \leq \rho(m)+\sum_{i=0}^{-1+\rho(m)} \mu\left(m, \alpha_{i}\right) .
$$

From the facts that:

- $\sum_{f=1}^{h} v^{\alpha_{i}}(h-f) \leq \mu\left(m, \alpha_{i}\right)$

- $\forall n \geq h, v^{\alpha_{i}}(n)=0$

- $\{w(n, d): n \geq 0\}$ is the shuffle of $d+1$ subsequences of the form $\left\{v^{\alpha_{i}}(n)\right.$ : $n \geq 0\}$ and of $\rho(m)-d-1$ subsequences of the form $\left\{x^{\alpha_{i}}(n): n \geq 0\right\}$ 
we deduce that:

$$
\sum_{i=d+1}^{-1+\rho(m)} \mu\left(m, \alpha_{i}\right) \leq \sum_{f=1}^{h} w(n-f, d) \leq-d-1+\rho(m)+\sum_{i=0}^{-1+\rho(m)} \mu\left(m, \alpha_{i}\right) .
$$

\section{Proof of Proposition 3}

The state of the neuron $z$ at time $n$ is given by the formula:

$$
\begin{aligned}
z(n, d) & =\mathbf{1}\left[\sum_{f=1}^{h} c(f, d) z(n-f, d)-\theta_{2}(d)\right] \\
& =\mathbf{1}\left[\sum_{1 \leq f \leq h, f \notin A(d)} c(f, d) z(n-f, d)+\sum_{f \in A(d)} c(f, d) z(n-f, d)-\theta_{1}-\xi(d)\right] \\
& =\mathbf{1}\left[\sum_{1 \leq f \leq h, f \notin A(d)} b_{f} z(n-f, d)+\sum_{f \in A(d)} b_{f} z(n-f, d)+Q 2(n, d)-\theta_{1}\right]
\end{aligned}
$$

By hypothesis, we known that $n-f \equiv 0(\bmod \rho(m))$ implies that $z(n-$ $f, d)=y(n-f)$. From Equation (37), we have $f \not \equiv 0(\bmod \rho(m))$ implies that $b_{f}=0$. Consequently, it follows that:

$$
\begin{aligned}
z(n, d) & =\mathbf{1}\left[\sum_{1 \leq f \leq h, f \equiv 0(\bmod \rho(m))} b_{f} y(n-f)+Q 2(n, d)-\theta_{1}\right] \\
& =\mathbf{1}\left[S 2(n)+Q 2(n, d)-\theta_{1}\right]
\end{aligned}
$$

\section{Proof of Proposition 4}

By hypothesis, we have $\sum_{f \in A(d)} z(n-f, d)=\operatorname{Tot}(d)$. It follows that:

$$
\begin{aligned}
\beta(d) \sum_{f \in A(d)} z(n-f, d) & =\beta(d) \times \operatorname{Tot}(d) \\
& =\lambda .
\end{aligned}
$$


We deduce that: $Q 2(n, d)=\frac{\beta(d)}{8}$.

\section{Proof of Proposition [5}

By hypothesis, we have $\sum_{f \in A(d)} z(n-f, d) \leq \operatorname{Tot}(d)-1$. It follows that

$$
\begin{gathered}
\beta(d) \times(\operatorname{Tot}(d)-1) \leq \beta(d) \sum_{f \in A(d)} z(n-f, d) \\
\beta(d) \times(\operatorname{Tot}(d)-1)-\xi(d) \leq \beta(d) \sum_{f \in A(d)} z(n-f, d)-\xi(d) .
\end{gathered}
$$

We obtain:

$$
\frac{-7 \beta(d)}{8} \leq Q 2(n, d)
$$

Likewise, we have : $0 \leq \sum_{f \in A(d)} z(n-f, d)$. It follows that :

$$
\begin{aligned}
\beta(d) \sum_{f \in A(d)} z(n-f, d) & \leq 0 \\
\beta(d) \sum_{f \in A(d)} z(n-f, d)-\xi(d) & \leq-\xi(d) \\
Q 2(n, d) & \leq-\xi(d) .
\end{aligned}
$$

We deduce that: $\frac{-7 \beta(d)}{8} \leq Q 2(n, d) \leq-\xi(d)$.

\section{Proof of Lemma 15}

The proof is subdivided into two parts:

First part:

Based on the facts that:

- $0 \leq d \leq-1+\rho(m) \leq 2 m \leq p_{i}$, for i such that $0 \leq i \leq-1+\rho(m)$

- From Equation (13), we deduce that $\rho(m) \geq 2$ implies that $\mu\left(m, \alpha_{i}\right) \geq 4$

- the fact that $\{y(n): n \geq 0\}$ is the shuffle of $\rho(m)$ subsequences $\left\{x^{\alpha_{i}}(n)\right.$ : $n \geq 0\}$ 
- the structure of the subsequence $\left\{x^{\alpha_{i}}(n): n \geq 0\right\}$ defined in Equation (14)

we easily deduce that it is possible by using only $B_{0}(d), p_{0}, p_{1}, \ldots, p_{-1+\rho(m)}$ to construct all the terms

$y(j)$ such that $1-\rho(m)+L_{1}(d) \leq j \leq-\rho(m)+h+L_{1}(d)$.

Second part:

The proof will be done by contradiction. Let us suppose that :

$$
\begin{gathered}
n \not \equiv h-\rho(m)+L_{1}(d) \quad\left(\bmod L_{2}\right) \\
\sum_{f \in B_{0}(d)} y(n-f)=\operatorname{Tot}(d)
\end{gathered}
$$

Based on the First part, on Equation (48), on the period of the sequence $\{y(n)$ : $n \geq 0\}$, we deduce that

$$
n \equiv h-\rho(m)+L_{1}(d) \quad\left(\bmod L_{2}\right)
$$

This implies a contradiction with Equation (81).

\section{Proof of Lemma 16}

Based on:

- the definition of the set $A(d)$

- the construction of the set $A(d)$ from $B_{i}(d) ; 0 \leq i \leq d$

- the Lemma 15

we deduce the result. 


\section{Proof of Lemma 17}

The proof will be done by induction on $t$ :

Basic Case: steps $0 \leq t \leq h-1$

The result can be deduced from Equation (157).

Induction hypothesis: we suppose the property is true at all steps $n-1$ such that $h-1 \leq n-1 \leq L_{1}(d)+h-2-\rho(m)$.

\section{Step $n$.}

Firstly, we want to show that $\sum_{f \in A(d)} y(n-f) \leq \operatorname{Tot}(d)-1$.

From induction hypothesis, we easily deduce that: $n \leq L_{1}(d)+h-1-\rho(m)$.

By application of Lemma [16, we conclude that:

$$
\sum_{f \in A(d)} y(n-f) \leq \operatorname{Tot}(d)-1 .
$$

Consequently, by applying Proposition [5 we deduce that

$$
\frac{-7 \beta(d)}{8} \leq Q 2(n, d) \leq-\xi(d)
$$

Secondly, we want to show that $z(n, d)=y(n)$. From the induction hypothesis and Proposition 3, we know that: $z(n, d)=\mathbf{1}\left[S 2(n)+Q 2(n, d)-\theta_{1}\right]$. Given the value of $S 2(n)$, we shall distinguish two cases:

Case $1: S 2(n) \geq \theta_{1}$

This implies that $y(n)=1$. From Equation (83), we have $0<Q 2(n)$. It follows that:

$$
0<\frac{-7 \beta(d)}{8} \leq S 2(n)+Q 2(n, d)-\theta_{1} .
$$

Consequently: $z(n, d)=1=y(n)$.

Case $2: S 2(n)<\theta_{1}$

This implies that $y(n)=0$. From Remark 3, we have $S 2(n)-\theta_{1} \leq \lambda$. From Equation (83), we have $Q 2(n, d) \leq-\xi(d)$. It follows that $S 2(n)+Q 2(n, d)-$ $\theta_{1} \leq \lambda-\xi(d)<0$. Consequently $z(n, d)=0=y(n)$. 


\section{Proof of Lemma 18}

From Equation (35), Lemma 5 and Lemma 6, it is easy to deduce that:

$$
y(t)=1 \text { i.e. } S 2(t)=\theta_{1} \text { for } \mathrm{t} \text { such that } h-\rho(m) \leq t \leq h-\rho(m)+d .
$$

Based on the periods of the subsequences $\left\{x^{\alpha_{i}}(n): n \geq 0\right\}$, we conclude that:

$y(t)=1$ i.e. $S 2(t)=\theta_{1}$ for $\mathrm{t}$ such that $L_{1}(d)+h-\rho(m) \leq t \leq L_{1}(d)+h-\rho(m)+d$

From:

- the definition of the sets $B_{i}(d)(0 \leq i \leq d)$ and $A(d)$

- Proposition 4

we conclude that:

$Q 2(t, d)=\frac{\beta(d)}{8}$ for $\mathrm{t}$ such that $L_{1}(d)+h-\rho(m) \leq t \leq L_{1}(d)+h-\rho(m)+d$

From Proposition 3 and Lemma 17, we deduce that:

$$
z(t, d)=\mathbf{1}\left[S 2(t)+Q(t, d)-\theta_{1}\right]
$$

From Equation (85), Equation (86) and Equation (87), we conclude that:

$$
\begin{aligned}
z(t, d) & =\mathbf{1}\left[\theta_{1}+\frac{\beta(d)}{8}-\theta_{1}\right] \\
& =\mathbf{1}\left[\frac{\beta(d)}{8}\right] \\
& =0
\end{aligned}
$$

\section{Proof of Lemma 19}

From Proposition 5. Lemma 16, Lemma 17 and Lemma 18, we deduce that:

$$
\frac{-7 \beta(d)}{8} \leq Q 2(t, d) \leq-\xi(d) .
$$


From Proposition 3, we have:

$$
z(t, d)=\mathbf{1}\left[S 2(t)+Q 2(t, d)-\theta_{1}\right] .
$$

Based on the value of $S 2(t)$, we can distinguish two cases:

First Case: $S 2(t)=\theta_{1}$

If follows that

$$
0 \leq \frac{-7 \beta(d)}{8} \leq S 2(t)+Q 2(t, d)-\theta_{1}
$$

Consequently

$$
z(t, d)=y(t)=1
$$

Second Case: $S 2(t)<\theta_{1}$

Based on Remark 3 and Equation (88), it follows that:

$$
S 2(t)+Q 2(t, d)-\theta_{1} \leq \lambda-\xi(d)<0
$$

Consequently

$$
z(t, d)=y(t)=0
$$

\section{Proof of Lemma 20}

The proof will be done by induction on $t$.

\section{Basic Case:}

Induction hypothesis: assuming that:

$$
\begin{aligned}
& z\left(h+L_{1}(d)-i, d\right)=w(h-i, d) \text { for } i \text { such that } 1 \leq i \leq h \\
& z\left(h+L_{1}(d)+j, d\right)=w(h+j, d) \text { for } j \text { such that } 0 \leq j \leq \ell-1
\end{aligned}
$$

Step $\ell$ : 


$$
\begin{aligned}
z\left(\ell+h+L_{1}(d), d\right) & =\mathbf{1}\left[\sum_{f=1}^{h} c(f, d) z\left(-f+\ell+h+L_{1}(d), d\right)-\theta_{2}(d)\right] \\
& =\mathbf{1}\left[\sum_{f=1}^{h} b_{f} z\left(-f+\ell+h+L_{1}(d), d\right)-\theta_{1}+Q 2\left(\ell+h+L_{1}(d), d\right)\right] .
\end{aligned}
$$

From (a) of Lemma 10, Lemma 17, Lemma 18 and Lemma 19 we have:

$$
\sum_{f \in A(d)} z\left(-f+\ell+h+L_{1}(d), d\right) \leq \sum_{f \in A(d)} y\left(-f+\ell+h+L_{1}(d)\right) .
$$

From Proposition 5. Lemma 16 and Equation (93) we deduce that

$$
\frac{-7 \beta(d)}{8} \leq Q 2\left(\ell+h+L_{1}(d), d\right) \leq-\xi(d) .
$$

From Equation (92), by hypothesis defined by Equation (89) and by hypothesis defined by Equation (90), we can write:

$$
\begin{aligned}
z\left(\ell+h+L_{1}(d), d\right) & =\mathbf{1}\left[\sum_{f=1}^{h} b_{f} w(\ell+h-f, d)-\theta_{1}+Q 2\left(\ell+h+L_{1}(d), d\right)\right] \\
& =\mathbf{1}\left[S 3(\ell+h, d)-\theta_{1}+Q 2\left(\ell+h+L_{1}(d), d\right)\right]
\end{aligned}
$$

The proof will be subdivided into two cases:

First Case: $\ell \equiv \jmath(\bmod \rho(m))$ and $\jmath \in\{0,1,2, \ldots, d\}$

By basing ourselves on the part (a) of Lemma 10, Remark 5 , Lemma 17, Lemma 18 and Lemma 19, we deduce that:

$$
S 3(\ell+h, d)-\theta_{1} \leq-2 .
$$

By using Equation (94), we deduce that:

$$
S 3(\ell+h, d)-\theta_{1}+Q 2\left(\ell+h+L_{1}(d), d\right) \leq-2-\xi(d)<0
$$

It follows that:

$$
z\left(\ell+h+L_{1}(d), d\right)=0=w(h+\ell, d)
$$


Second Case: $\ell \equiv \jmath(\bmod \rho(m))$ and $\jmath \in\{d+1, d+2, \ldots,-1+\rho(m)\}$

Let us note $\ell=(\imath \times \rho(m))+\jmath$ where $\imath \in \mathbb{N}$ and $\jmath \in\{d+1, d+2, \ldots,-1+\rho(m)\}$.

Based on the structure of $b_{f}$ (see Equation (37)) and on Remark 5, we deduce that:

$$
S 3(\ell+h, d)=\sum_{f=1}^{k} \bar{a}_{f} x^{\alpha_{\jmath}}\left(k+1+\gamma_{\jmath}(d)+\imath-f\right)
$$

Based on the value of $S 3(\ell+h, d)$, we have to distinguish two subcases:

Subcase 1: $S 3(\ell+h, d)=\theta_{1}$

The hypothesis $S 3(\ell+h, d)=\theta_{1}$ implies that $w(h+\ell, d)=1$. From Equation (94) we deduce that:

$$
0 \leq \frac{-7 \times \beta(d)}{8} \leq S 3(\ell+h, d)-\theta_{1}+Q 2\left(\ell+h+L_{1}(d), d\right)
$$

It follows that:

$$
z\left(\ell+h+L_{1}(d), d\right)=1=w(h+\ell, d)
$$

Subcase 2: $S 3(\ell+h, d)<\theta_{1}$

The hypothesis $S 3(\ell+h, d)<\theta_{1}$ implies that $w(h+\ell, d)=0$. From Remark 3 and the hypothesis, we deduce that:

$$
S 3(\ell+h, d)-\theta_{1} \leq \lambda
$$

From Equation (94) we deduce that:

$$
S 3(\ell+h, d)-\theta_{1}+Q 2\left(\ell+h+L_{1}(d), d\right) \leq \lambda-\xi(d)<0
$$

It follows that:

$$
z\left(\ell+h+L_{1}(d), d\right)=0=w(h+\ell, d) .
$$

\section{Proof of Lemma 21}

The proof shall be done by a strong induction on $t$.

Basic Case: steps $0,1,2, \ldots, h-1$

With regards: 
- to the configuration of the sequence $\{z(n, d): n \geq 0\}$ at the end of phase 4 (Remark 7)

- the transient length of the sequence $\{w(n, d): n \geq 0\}$

- the initial configuration of the sequence $\{w(n, d): n \geq 0\}$ (see Equations (39) and (40))

we deduce that

$z\left(f+L_{4}(d), d\right)=w\left(f+h+d+1+\left(\rho(m) \times\left(-1-p_{d}\right)\right), d\right) ; \forall f \in \mathbb{N}, 0 \leq f \leq h-1$

Induction hypothesis: steps $\ell-h, \ell-h+1, \ldots, \ell-1$

We suppose that the property holds for all $\jmath$ such that $\ell-h \leq \jmath \leq \ell-1$, i.e.,

$$
z\left(\jmath+L_{4}(d), d\right)=w\left(\jmath+h+d+1+\left(\rho(m) \times\left(-1-p_{d}\right)\right), d\right)
$$

Step $\ell$ : It is clear that

$$
\ell \geq h
$$

We also have:

$$
\begin{aligned}
z\left(\ell+L_{4}(d), d\right) & =\mathbf{1}\left[\sum_{f=1}^{h} c(f, d) z\left(-f+\ell+L_{4}(d), d\right)-\theta_{2}(d)\right] \\
& =\mathbf{1}\left[\sum_{f=1}^{h} b_{f} z\left(-f+\ell+L_{4}(d), d\right)-\theta_{1}+Q 2\left(\ell+L_{4}(d), d\right)\right] \\
& =\mathbf{1}\left[\sum_{f=1}^{h} b_{f} w\left(-f+\ell+h+d+1+\left(\rho(m) \times\left(-1-p_{d}\right)\right), d\right)-\theta_{1}+Q 2\left(\ell+L_{4}(d), d\right)\right] \\
& =\mathbf{1}\left[S 3\left(\ell+h+d+1+\left(\rho(m) \times\left(-1-p_{d}\right)\right), d\right)-\theta_{1}+Q 2\left(\ell+L_{4}(d), d\right)\right]
\end{aligned}
$$

From Equation (97), we have $\ell \geq h$. By using Lemma 16, we deduce that:

$$
\sum_{f \in A(d)} y\left(-f+\ell+L_{4}(d)\right) \leq \operatorname{Tot}(d)-1
$$


From Lemma 17] Lemma 18, Lemma 19] and Lemma 20, we easily deduce that:

$$
\sum_{f \in A(d)} z\left(-f+\ell+L_{4}(d), d\right) \leq \sum_{f \in A(d)} y\left(-f+\ell+L_{4}(d)\right)
$$

From Equations (102) and (103), we easily deduce that:

$$
\sum_{f \in A(d)} z\left(-f+\ell+L_{4}(d), d\right) \leq \operatorname{Tot}(d)-1
$$

Consequently by applying Proposition [5, we deduce that:

$$
\frac{-7 \beta(d)}{8} \leq Q 2\left(\ell+L_{4}(d), d\right) \leq-\xi(d) .
$$

Let us suppose that $\ell=\left(i_{1} \times \rho(m)\right)+i_{2}$ with $0 \leq i_{2} \leq-1+\rho(m)$. We have to distinguish two cases:

First Case: $0 \leq i_{2} \leq \rho(m)-d-2$

Based on the value of $S 3\left(\ell+h+d+1+\left(\rho(m) \times\left(-1-p_{d}\right)\right), d\right)$, we have to distinguish two subcases:

Subcase 1: $S 3\left(\ell+h+d+1+\left(\rho(m) \times\left(-1-p_{d}\right)\right), d\right)=\theta_{1}$

The hypothesis $S 3\left(\ell+h+d+1+\left(\rho(m) \times\left(-1-p_{d}\right)\right), d\right)=\theta_{1}$ implies that $w\left(\ell+h+d+1+\left(\rho(m) \times\left(-1-p_{d}\right)\right), d\right)=1$. From Equation (105), we deduce that:

$0 \leq \frac{-7 \beta(d)}{8} \leq S 3\left(\ell+h+d+1+\left(\rho(m) \times\left(-1-p_{d}\right)\right), d\right)-\theta_{1}+Q 2\left(\ell+L_{4}(d), d\right)$

It follows that

$$
z\left(\ell+L_{4}(d), d\right)=1
$$

i.e. $z\left(\ell+L_{4}(d), d\right)=w\left(\ell+h+d+1+\left(\rho(m) \times\left(-1-p_{d}\right)\right), d\right)$.

$$
\text { Subcase 2: } S 3\left(\ell+h+d+1+\left(\rho(m) \times\left(-1-p_{d}\right)\right), d\right)<\theta_{1}
$$

The hypothesis $S 3\left(\ell+h+d+1+\left(\rho(m) \times\left(-1-p_{d}\right)\right), d\right)<\theta_{1}$ implies that $w\left(\ell+h+d+1+\left(\rho(m) \times\left(-1-p_{d}\right)\right), d\right)=0$. From Remark 3 and the hypothesis, 
we deduce that:

$$
S 3\left(\ell+h+d+1+\left(\rho(m) \times\left(-1-p_{d}\right)\right), d\right)-\theta_{1} \leq \lambda
$$

From Equation (105) we deduce that:

$S 3\left(\ell+h+d+1+\left(\rho(m) \times\left(-1-p_{d}\right)\right), d\right)-\theta_{1}+Q 2\left(\ell+L_{4}(d), d\right) \leq \lambda-\xi(d)<0$

It follows that:

$$
z\left(\ell+L_{4}(d), d\right)=0=w\left(\ell+h+d+1+\left(\rho(m) \times\left(-1-p_{d}\right)\right), d\right) .
$$

Second Case: $\rho(m)-d-1 \leq i_{2} \leq \rho(m)-1$

From induction hypothesis and Remark 7, it is clear that:

$$
\sum_{f=1}^{h} b_{f} w\left(-f+\ell+h+d+1+\left(\rho(m) \times\left(-1-p_{d}\right)\right), d\right)=0
$$

Consequently, we have:

$$
w\left(\ell+h+d+1+\left(\rho(m) \times\left(-1-p_{d}\right)\right), d\right)=0 .
$$

Based on Equation (105) and on Equation (106), we deduce that:

$S 3\left(\ell+h+d+1+\left(\rho(m) \times\left(-1-p_{d}\right)\right), d\right)-\theta_{1}+Q 2\left(\ell+L_{4}(d), d\right) \leq-\theta_{1}-\xi(d)<0$

From Equation (101), we deduce that:

$$
z\left(\ell+L_{4}(d), d\right)=0
$$

It follows that:

$$
w\left(\ell+h+d+1+\left(\rho(m) \times\left(-1-p_{d}\right)\right), d\right)=z\left(\ell+L_{4}(d), d\right) .
$$




\section{Proof of Lemma 22}

The result is deduced from the fact that the transient phase finishes at time $L_{4}(d)$ and from the fact that the period of the sequence $\{w(n, d): n \geq 0\}$ is $L_{0}(d)$.

\section{Proof of Lemma 23}

Based on Equation (37), on Equation (58) and from the following hypothesis:

$$
d<\beta\left(m, \alpha_{e}\right)
$$

we easily deduce that:

$$
c(f, d)=0 \text {; for } f \text { such that } h-\beta\left(m, \alpha_{e}\right)+d+1 \leq f \leq h .
$$

From one of the following initial configurations $y(0) y(1) \ldots y(h-1)$ or $\widetilde{z}(0, d) \widetilde{z}(1, d) \ldots \widetilde{z}(h-$ $1, d)$, the neuronal recurrence equation $\{z(n, d): n \geq 0\}$ converges to the same basin of attraction because

$$
\widetilde{z}(\ell, d)=y(\ell) \text { for } \ell \text { such that } \beta\left(m, \alpha_{e}\right)-d \leq \ell \leq h-1
$$

and

$$
c(f, d)=0 ; \text { for } f \text { such that } h-\beta\left(m, \alpha_{e}\right)+d+1 \leq f \leq h .
$$

\section{References}

[1] E. Goles and S. Martínez, "Neural and Automata Networks, Dynamical Behaviour and Applications" (Norwell, MA, Kluwer Academic Publishers, 1990)

[2] S. Haykin, "Neural Networks : A Comprehensive Foundations" (Upper Saddle River, New Jersey : Prentice-Hall, 1999) 
[3] E. R. Caianiello and A. De Luca, "Decision Equation for Binary Systems : Applications to Neuronal Behavior," Kybernetic, 3(1966) 33-40

[4] M. Cosnard, D. Moumida, E. Goles and T.de.St. Pierre, "Dynamical Behavior of a Neural Automaton with Memory," Complex Systems, 2, (1988), 161-176.

[5] M. Cosnard, M. Tchuente and G. Tindo, "Sequences Generated by Neuronal Automata with Memory", Complex Systems, 6,1992, 13-20.

[6] M. Cosnard and E. Goles, "Dynamique d'un automate à mémoire modélisant le fonctionnement d'un neurone", Comptes Rendus de l'Académie des Sciences, tome 299, Série I, n10, 459-461, 1984.

[7] W. S. McCulloch and W. Pitts, "A Logical Calculus of the Ideas Immanent in Nervous Activity", Bulletin of Mathematical Biophysics, 5 (1943) 115-133.

[8] E. Goles and S. Martínez, "Exponential Transient Classes of Symmetric Neural Networks for Synchronous and Sequential Updating", Complex Systems 3(1989) 589-597.

[9] Hopfield, J. J., "Neural Networks and Physical Systems with Emergent Collective Computational Abilities", Proc. Nat. Acad. Sci. U.S.A 79 1982, 25542558.

[10] Hopfield, J. J., D.W. Tank, "Neural Computation of Decision in Optimization Problems ", Biol. Cybern., 52 1985, 141-152.

[11] R. Ndoundam and M. Matamala, "Cyclic Evolution of Neuronal Automata with Memory When All the Weighting Coefficients are Strictly Positive", Complex Systems, 12(2000), 379-390.

[12] R. Ndoundam and M. Matamala, "No Polynomial Bound for the Period of Neuronal Automata with Memory With Inhibitory Memory", Complex Systems, 12(2000), 391-397. 
[13] R. Ndoundam and M. Tchuente, "Exponential transient length generated by a neuronal recurrence equation", Theoretical Computer Science, 306(2003), 513-533.

[14] R. Ndoundam and M. Tchuente, "Exponential Period of Neuronal Recurrence Automata with Excitatory Memory", Complex Systems, 15(2004), 79-88.

[15] M. Tchuente and G. Tindo, "Suites générées par une équation neuronale à mémoire",Comptes Rendus de l'Académie des Sciences, tome 317, Serie I, 625-630, 1993.

[16] J. Šima, P. Orponen and T. Anti-Poika, "On the computational Complexity of Binary and Analog Symmetric Hopfield Nets",Neural Computation, 12, 2965-2989, 2000.

[17] J. S̆ima and P. Orponen "A continuous-time Hopfield net simulation of discrete neural networks ", in Proceedings of NC'2000:Second International ICSC Symposium on Neural Computing, pages 36-42, Wetaskiwin, Canada:ICSC Academic Press.

[18] M. Cosnard, "Dynamic Properties of An Automaton with Memory", pages 293-302 in Cellular Automata : A Parallel Model, edited by M. Delorme and J. Mazoyer (Kluwer Academics Publishers, 1999).

[19] F. Fogelman Soulié, and al., "Automata Networks and Artificial Intelligence," pages 133-186 in Automata Networks in Computer Science : Theory and Applications edited by F. Fogelman, Y. Robert, and M. Tchuente (Manchester, Manchester University Press, 1987).

[20] D. Moumida, "Contribution à l'étude de la Dynamique d'un Automate à Mémoire," ( Doctoral dissertation, University of Grenoble, 1989). 